\title{
Experimental Study of Anode Shape Prediction in Through Mask Electrochemical Micro Machining
}

\author{
P. Jain ${ }^{1 *}$, D. Gehlot ${ }^{2}$,T. Bagoriya ${ }^{3}$ \\ ${ }^{1}$ Assitant Professor, Aryabhatta College of engineering \& research Center Ajmer,India \\ ${ }^{2}$ Assitant Professor, Govt Engineering College Ajmer, India \\ ${ }^{3}$ PG Student, Govt Engineering College Ajmer, India \\ \{pankajjainksg@gmail.com\}
}

\begin{abstract}
To manufacture quality products at lowest cost in industries, optimization is an effective technique which can be Electrochemical micro-machining (EMM) appears to be very promising as a future micromachining technique, since in many areas of applications it offers several advantages, which include higher machining rate, better precision and control, short lead time and a wide range of materials that can be machined.In this work, the shape evolution in through-mask electrochemical micromachining (ECMM) process is investigated numerically and experimentally. The effect of process parameters on the anode shape was demonstrated by Finite Element Method using COMSOL Multiphysics software. With finite element method (FEM), the anodic evolution process is predicted and effects of stray current also have been identified. The validation experiment is conducted and the hole drilling procedure is observed. The FEM calculation predicted model is in good agreement with experimental model. With the sets of experiments and Finite element analysis simulation, the optimized set and the formulation of the electrolyte in through-mask ECMM are achieved.
\end{abstract}

Keywords: Electrochemical micromachining, Tooling Design, anode shape prediction, COMSOL Tool

\section{Introduction}

Since miniaturization will continue as long as people require efficient space utilization with more efficient and better quality product, micromachining technology will become still more important in the future. ECMM is an electrochemical reaction of the anodic dissolution process. It is the reverse of electroplating. The several advantages of ECM is no tool wear, stress-free, high throughput, smooth surfaces, and the ability to machine complex shapes in materials regardless of their hardness or whether they are heat-resistant materials[1]. In through-mask EMM, a photo resist patterned metal workpiece is made an anode in an electrochemical cell such that the exposed metal surface is removed by high-rate anodic metal dissolution by passing an external current. Compared with maskless EMM, through-mask EMM offers higher throughput and smaller pattern fabrication ability due to its parallel process in nature and no superfine electrode requirement [2].

Metal removal rate (MRR) or simulation in electrochemical machining (ECM) depends upon a large number of highly interrelated parameters such as electric field distribution, electrolyte conductivity $(\mathrm{k})$, temperature $(\mathrm{T})$, valency of dissolution $(\mathrm{Z})$, electrolyte flow velocity $(\mathrm{V})$ etc. Thus ECM is a fairly complex process which involves the simultaneous occurrence of two phase fluid dynamics, conjugate heat transfer, mass transfer, thermodynamics, and electrochemistry between moving boundaries. [3] For instance the electrolyte flow field affects the potential distribution, which affects the electrode reaction rate and thus the workpiece shape evolution which once again changes flow field. For this reason, modeling and simulating ECM is a very interesting topic. If the goal of a complete ECM simulation which incorporates all of these aspects is to be achieved, a thorough understanding of the fundamental processes and their interactions is mandatory.

In earlier "trial and error" methods with machining conditions is used to obtain the correct tool geometry. Such methods are expensive, time consuming, and have low accuracy.

B. Iyer, S. Nalbalwar and R. Pawade (Eds.)

ICCASP/ICMMD-2016. Advances in Intelligent Systems Research.

Vol. 137, Pp. 220-228.

(C) 2017. The authors - Published by Atlantis Press

This is an open access article under the CC BY-NC license (http://creativecommons.org/licens)es/by-nc/4.0/).

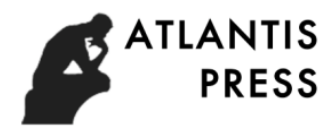


Analytic approaches are used for tooling design such as the $\cos \theta$ method [4,5], the complex variables method [6], and Semi-analytical and numerical techniques include the boundary elements method (BEM) [7-10], the finite difference method (FDM) [11-13], the finite volume method (FVM) and finite element method (FEM) $[14,15]$. The models used may greatly vary in terms of complexity.

This paper proposes a computer based simulation approach for anode shape profile evolution based on FEM using COMSOL multi physics tool in ECMM.

\section{Design tool}

Tool Design in ECM deals basically with the computation of tool shape which under specified machining conditions would produce a work piece having the prescribed shape and accuracy. For the prediction of anode (or work) shape obtainable from a tool while operating under the specified conditions of machining [19].

\subsection{Procedure for Anode shape prediction}

To analyse the variation in current density \& inter electrode gap (IEG) for any operating condition is a required for proper design of ECM tools, many of these anode shape prediction models are discussed in terms of the inter electrode gap.

The Matthias manufactured 3D geometrical micro structured by using jet-ECM for this COMSOL Multiphysics tool is used to simulate localized anodic dissolution process. The anode surface displacement depends on normal current density which followed by Faraday's law in the model to simulate the shape [16-18].

The V. K. Jain \& Dilip Gehlot had prepared a MATLAB model for prediction of shape evolving during electrochemical micro etching process which requires a moving boundary simulation where at each time step, the current distribution along the workpiece surface is calculated. Initially, the current density at the intersecting edge of the mask and substrate is maximum [20]. According to Tipton, tool design is based on the computation of IEG for the given operating conditions. In his theory he does not consider the effects of many important parameters such as the mode of electrolyte flow and change in electrical conductivity of the electrolyte.[21] Therefore, the exact path of the electric current flow lines within the IEG is difficult to determine analytically. This is one of the reasons responsible for the difference between the analytical and the experimental results. The following Laplace equation (1) has been solved for determining potential distribution in the IEG.

$\frac{\mathrm{d}^{2} \phi}{d X^{2}}+\frac{\mathrm{d}^{2} \phi}{\mathrm{dY}^{2}}=0$

From the potential distribution, the current density $(\mathrm{J})$ can be evaluated using $\operatorname{Eq}(2)$

$\mathrm{J}=\mathrm{K} \frac{\mathrm{d} \phi}{\mathrm{dn}}$

Where $\mathrm{K}$ is the conductivity of the electrolyte and $\mathrm{n}$ is the normal at a point on the work surface.

Step by step procedure followed for anode shape prediction is as follows [14]

- Decide whether the analysis to be made is one, two or three dimensional.

- Collect all the initial data in the proper units and, if necessary, preliminary computation (for example, flow velocity from the volumetric flow rate) is also done.

- Now, decide whether the problem is to be solved as a temperature distribution, or electric field potential distribution problem.

- The Electric field potential at different point is calculated by solving Laplace equation in one, two or three dimension as the case. This equation can be solved by one of the approximate numerical methods (FDM, FET, or BET).

From the above Electric field potential distribution, current density is calculated at different points

- Calculate the temperature at different point and is used to modify the electrolyte conductivity. Now IEG is computed at different point in the domain of interest so that expected anode shape can be predicted [19]. 


\section{Theoretical Model}

A 2D model of the process was created in COMSOL multi-physics software. A cross section of the tool, workpiece, and electrolyte geometry was taken and drawn in COMSOL. The electrolyte was assumed to be the space in between these boundaries.

\subsection{Problem Statement}

Fig. 1 represents schematically the shape evolution during electrochemical micromachining. In the fig, the pattern consists of a two-dimensional line. The thickness of the insulating mask is $0.1 \mathrm{~mm}$ and the width of the exposed surface $2 \mathrm{~mm}$. The metal thickness is considered as $1 \mathrm{~mm}$. The prediction of shape evolution during electrochemical etching requires a moving boundary simulation where, at each timed step, the current distribution along the metal surface is calculated.

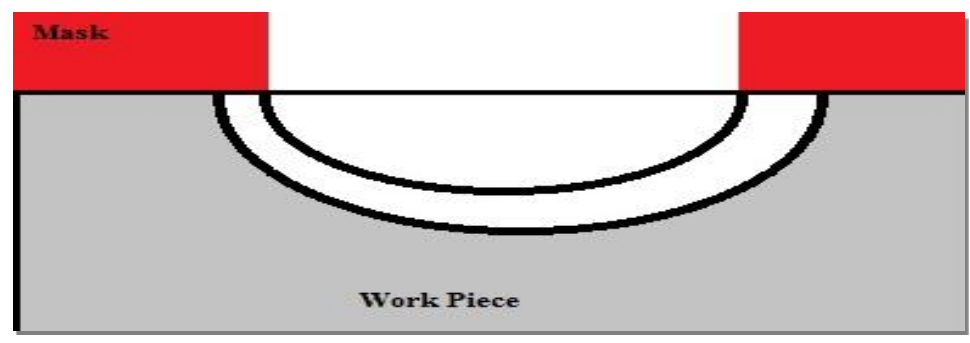

Fig. 1. Schematic view of shape evolution during ECM through mask

\subsection{Model Development}

The ECM model comprises several physical domains: Navier-Stokes flow description, electric field distribution, heat conduction \& convection, gas convection and moving boundaries (workpiece material removal). All constitute a multiphysics problem. Out of the real process an adequate geometry model needs to be deduced, which should be as simple as possible but comprises necessary process features according to the concrete goals of the simulation [22].

The modal developed for through-mask EMM, as shown in Fig. 2, consists of a conductive metal tool and an insulation layer. The mask is bonded to the anode. The metal tool serves as the cathode tool. The pink, green and red lines represent the cathode, anode boundaries and insulation layer respectively. The electrolyte flows onto the surface of the mask at a high speed and fills in all features in the metal and the insulation layer.

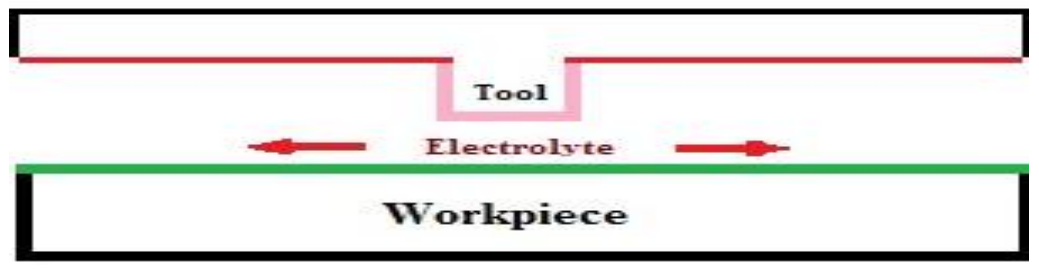

Fig. 2. Drawing of cross sectional model of machining process in COMSOL simulation

\subsection{Assumptions}

In the proposed micromachining, the current distribution defines the profile of micro anode shape evolution. Therefore, analysis of current density has been carried out. Modeling and simulation have been done to observe the current density distribution. The assumptions were made as follows:

The current density distribution at the anode surface is determined solely by the Ohmic effects.

The temperature $\mathrm{T} \&$ conductivity $\mathrm{k}$ of electrolyte is uniform.

Due to the ultrasonic agitation during process, the concentration gradient in the bulk electrolyte is negligible. 
Current efficient is constant during ECMM Process.

There is perfect adherence of masking on the anode surface and no current flow takes place through the masking.

Flow of electrolyte is assumed to be constant in the IEG and there would be no effect of changing the flow property in the material removal.

\subsection{Boundary Condition}

According to the electric field theory, the electric potential distribution in electrolyte is governed by Laplace's equation. The potential obeys Laplace's equation within gap domain CO1. A potential was applied to the anode boundary layers, and the cathode boundary layers were grounded. The side boundaries were insulated as shown in fig. 3. The electrolyte, the space separating the two electrodes, was given a constant conductivity value.

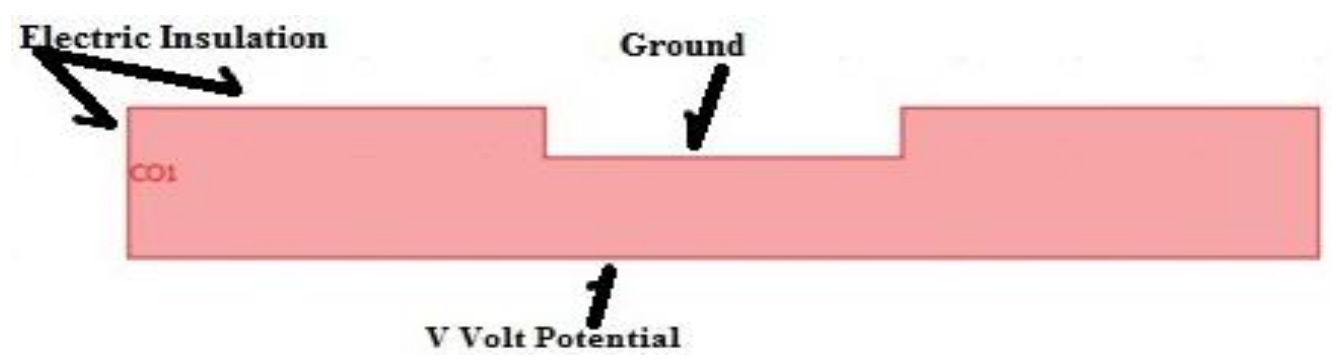

Fig. 3. Electric Potential Distribution Boundary Condition

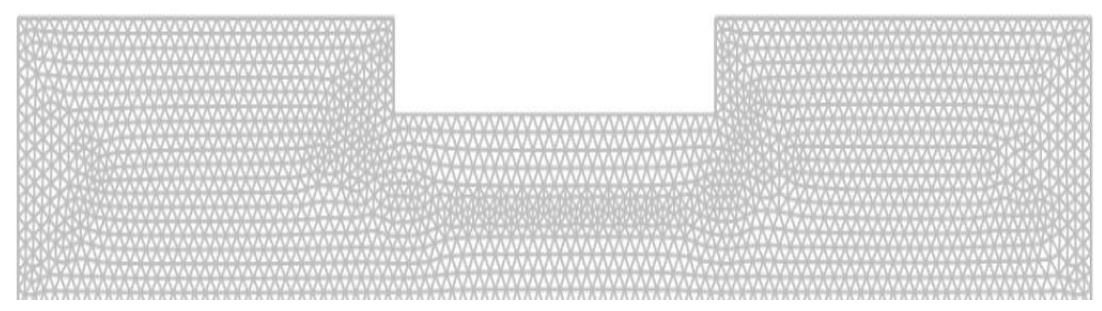

Fig. 4. Mesh Developed in simulation model

Table 1 Boundary conditions used in model

\begin{tabular}{|c|l|l|}
\hline Boundary & Condition & Defination \\
\hline 1 & $\mathrm{n}_{\mathrm{A}} \cdot J=0$ & Insulation \\
\hline 2 & $\varphi=10$ & Electric Potential \\
\hline 3 & $\mathrm{n}_{\mathrm{A}} \cdot J=0$ & Insulation \\
\hline 4 & $\varphi=0$ & Ground \\
\hline 5 & $\varphi=0$ & Ground \\
\hline 6 & $\varphi=0$ & Ground \\
\hline 7 & $\mathrm{n}_{\mathrm{A}} \cdot J=0$ & Insulation \\
\hline 8 & $\mathrm{n}_{\mathrm{A}} . J=0$ & Insulation \\
\hline
\end{tabular}

All electric boundary conditions are shown in table 1, where voltage is the electric potential, nA is the normal vector and $\mathrm{J}$ is the current density. The total voltage of $10 \mathrm{~V}$ over the working gap conforms the used power of the applied current supply, which has a maximum output of $2 \mathrm{~A}$.

\subsection{Electrodynamics \& Anodic Dissolution}

The application mode Conductive Media DC was used to define the electric parameters of the ECM process. In subdomain $\mathrm{CO} 1$, which represents the tool, work piece and electrolyte which is flowing in between both, an isotropic electric conductivity was defined with the experimental value of $4.6 \mathrm{~S} / \mathrm{m}$ or according to conductivity of electrolyte. 
Main driver of Electrochemical erosion is the anodic dissolution of metal bonds due to current density in normal direction Jn of process following Faraday's law. The removed material volume V is calculated by

$$
\mathrm{V}=\eta \cdot \frac{\mathrm{M}}{\rho \cdot \mathrm{Z} \cdot \mathrm{F}} \cdot \mathrm{J}_{\mathrm{n}}
$$

$\mathrm{M}$ is the molar mass, $\rho$ the density, $\mathrm{Z}$ the electrochemical valence of the material, $\mathrm{F}$ the Faraday constant, and $\eta$ is the current efficiency. Most influencing parameter for the material erosion is the electric field $\mathrm{E}$ since it affects the current density proportionately:

$$
\mathrm{J}=\sigma . \mathrm{E}
$$

A mesh displacement for boundary 2 was implemented following equations $\mathrm{Vn} \& \mathrm{~J}$.

Table 2 Variables in equation and used value for aluminum

\begin{tabular}{|c|l|l|}
\hline & Name & Value \\
\hline $\mathrm{H}$ & Current Efficiency & 1 \\
\hline $\mathrm{M}$ & Molar Mass & $26.98 \mathrm{gm} / \mathrm{mol}$ \\
\hline $\mathrm{Z}$ & Valancy & 3 \\
\hline$\rho$ & Mass Density & $2.70 \mathrm{gm} / \mathrm{m}^{3}$ \\
\hline $\mathrm{F}$ & Farady's Constant & $96500 \mathrm{C} / \mathrm{mol}$ \\
\hline
\end{tabular}

\section{Result and discussion}

The geometry of model is developed using drawing tool and then boundary condition is applied in which work surface \& tool surface is connected with positive and negative terminal of power supply respectively. This model discretized into small triangular elements which contains number of triangles, their nodes and edges. The Electric field potential at each node is calculated after solving Laplace equation with the help of simulation solver in COMSOL Tool. From the above Electric field potential distribution, current density is calculated at each node. The elements nodes are displaced by an amount equal to the linear material removed from each node which is calculated from current density at each node. After complication of first step, the gap between the tool to the newly generated surface of workpiece has been modified. Once the domain is changed, Laplace equation is solved again. The current density and amount of material removed are calculated after each time step. Geometry of the deformed surface obtained after iteration is computed and the loop continues. At each computational substep, the electrode surface was displaced proportionally to the current density according to Faraday's law of electrolysis and the machined profile was in turn used as the initial surface of the geometric model for current distribution computation in the next substep. The program flow chart for anode shape prediction using COMSOL multiphysics is shown in fig. 5.

Fig 6 (a) shows the tool and workpiece before machining while fig 6(b) shows the shape of the anode profile after machining. Fig 7 (a to c) shows the anode shape profile generated by COMSOL Multiphysics for different simulation time. it can be easily seen that as the machining time increases undercut increases. But after the certain value, the rate of undercut decreases because of decreasing the current density in the lateral direction. The shape evolution depends on the metal thickness, applied voltage, electrolyteconcentration and initial IEG.

\subsection{Simulation Model}

It can be seen that a 2D simulation of the anode shape profile of a single cavity subjected to anodic dissolution under control mass transport. The simulation involves the solving of Laplace equation for fixed concentration in a given time interval. The dissolved depth at each point is calculated from the local current density using Faraday's law and the procedure is repeated. 


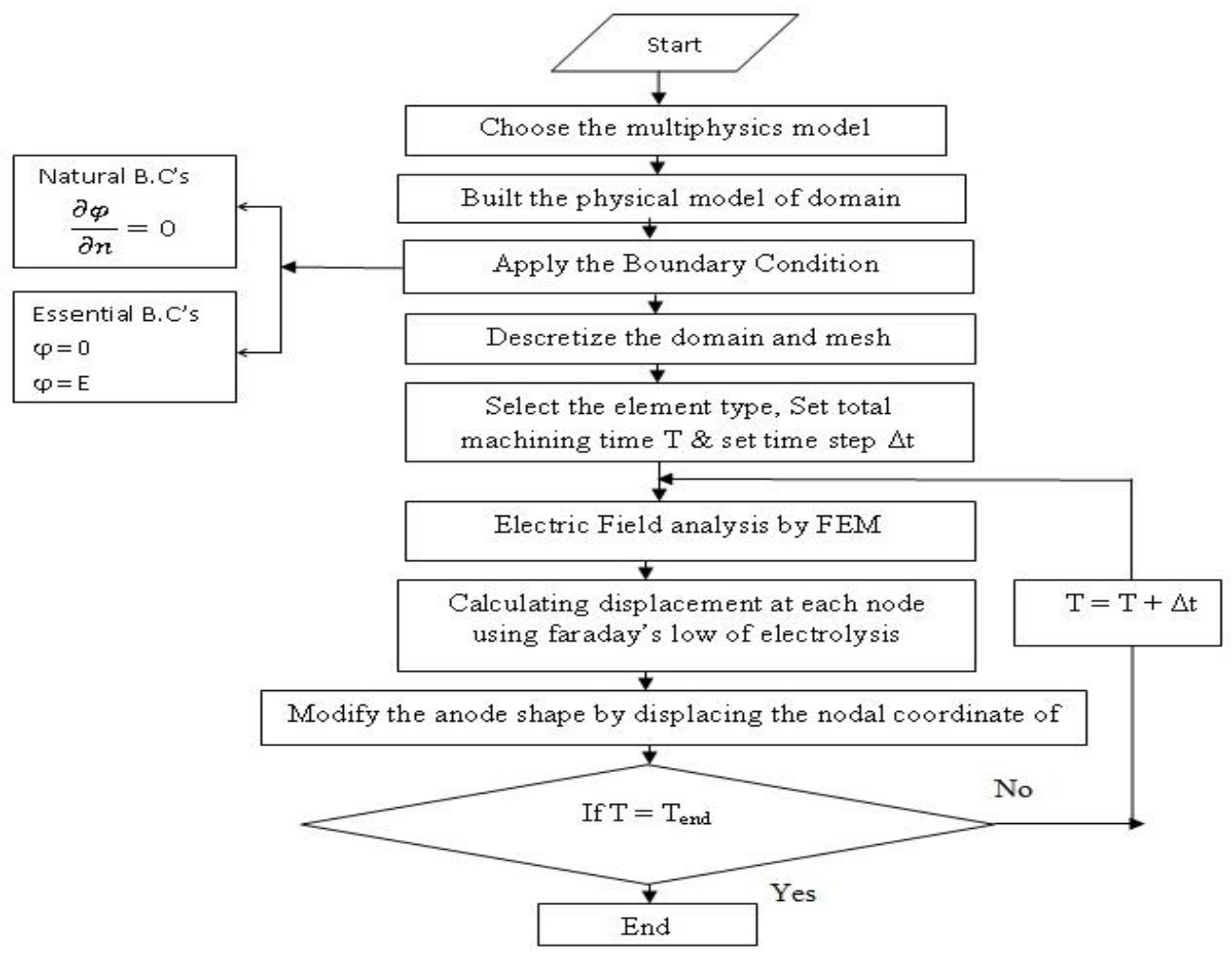

Fig. 5. Program flow chart for Anode Shape Prediction Using COMSOL

\section{Machining conditions}

Voltage $=10 \mathrm{~V}$, IEG $=0.2 \mathrm{~mm}$, Electrolyte Concentration $=40 \mathrm{gm} / \mathrm{lt}$, Work-Piece Material $=$ Aluminum \& Tool Material=Copper, work-piece having rectangular section $2 \times 2 \times 1 \mathrm{~mm}$ dimension, Insulation mask having $1 \times 1 \times 0.1$ $\mathrm{mm}$

It is seen that as the concentration and voltage increase, the current density is also increase which result in increase of electric current distribution field. Initially, the current distribution at metal surface is highly nonuniform and the maximum of the current density occurs at the edge of the mask. Since the metal removal rate is proportional to the current density, the maximum vertical displacement of the metal surface is away from the center of the hole. Initially the current density at the interfacing edge of the work piece and the mask is maximum and decreases towards the center.
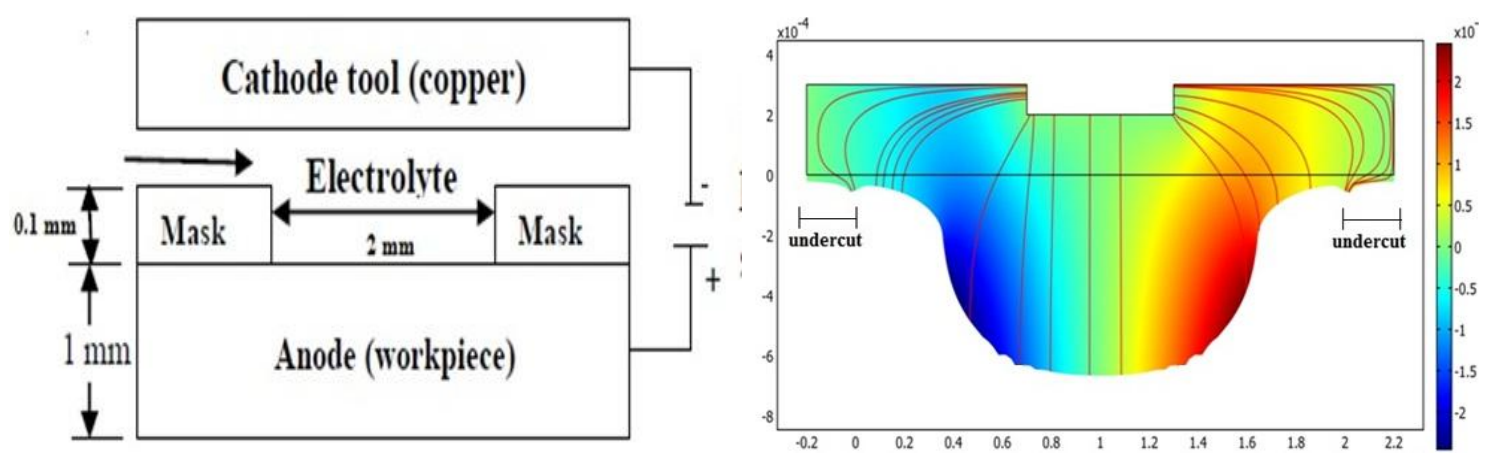

Fig. 6. (a) Intial Wprkpiece \& Tool Surface (b) Final Predicted anode Shape Profile for Through Mask ECMM at IEG $0.2 \mathrm{~mm} \mathrm{~V}=10$ volt 
As the machining proceeds, maximum current density shifts towards the center from the intersection edge which leads to reduce the amount of material removal in lateral direction, hence the amount of undercut in lateral direction reduces. The current density decreases with increasing the machining time because of increasing the gap as the tool feed is zero. It reduces the material removal in lateral and vertical direction. When the current distribution becomes stabilized, higher MRR is obtained at the center of the feature as compared to the edge. The higher current density at the edge of the feature is not desirable because it leads to higher lateral etching than that in the vertical direction.

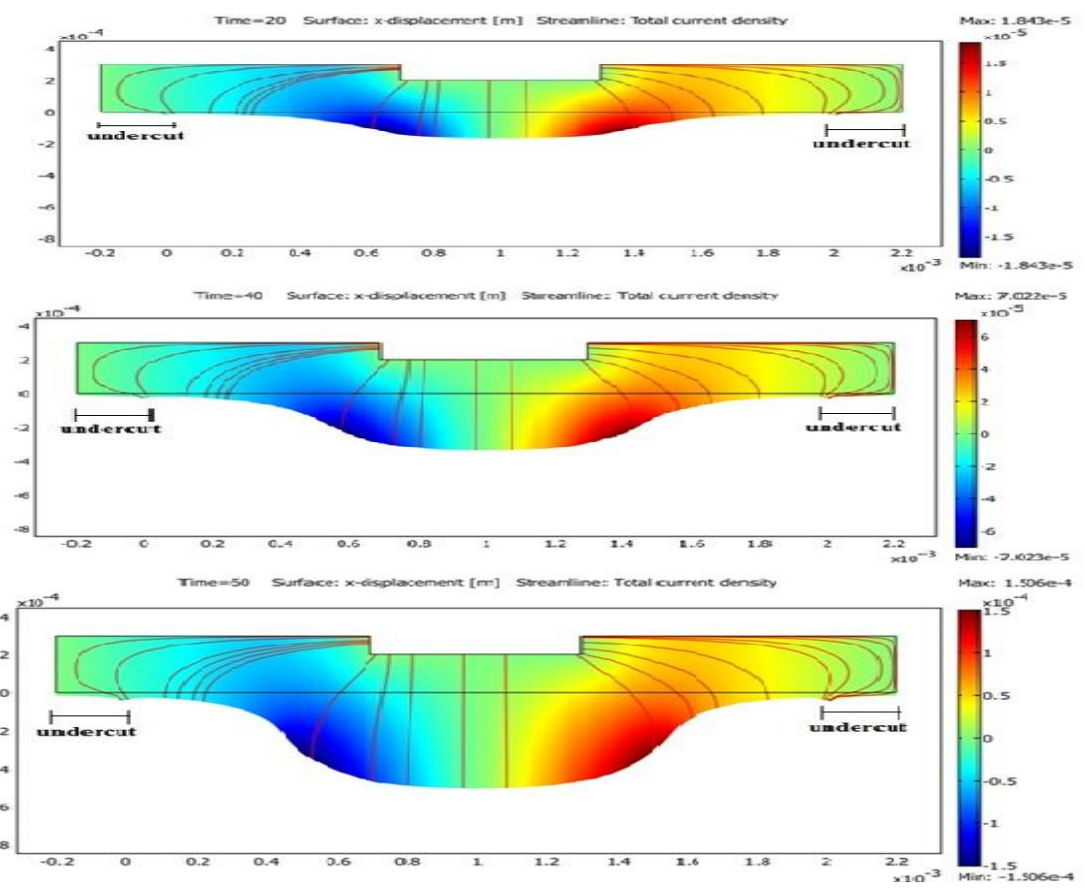

Fig. 7. (a to c) Predicted Anode Shape Profile in Through Mask ECMM

However, as the metal removal proceeds, the undercutting results in a redistribution of the current density at the substrate and the current distribution begins to shift from the edge to the center of the feature therefore the current density at the edge becomes low. From the results, it can be seen from formation of hole shape having parabolic or hemispherical shape.

\subsection{Validation of Simulation result}

To validate the simulation results, the shape evolution process is observed experimentally in which the sample with a insulating mask with thickness of $1 \mathrm{~mm}$ on aluminum sheet.

Hole produced by electro chemical micro machining is not throughly uniform. Time available for dissolution is more at the upper side of aluminum sheet, so more material is dissolved. The cross sectional cut of holes are shown in below fig. 8. It is seen from figure that initially localized current distribution leads to formation of hemispherical cross section view.
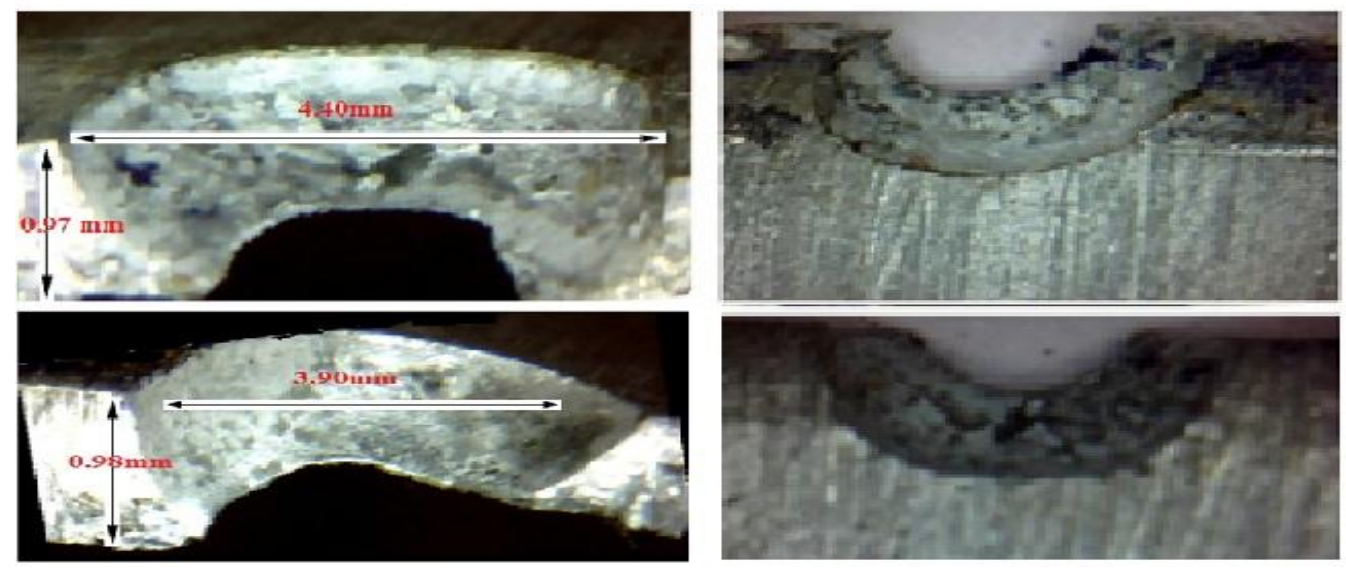

Fig. 8. Experimental Anode Shape Profile for Through Mask ECMM 


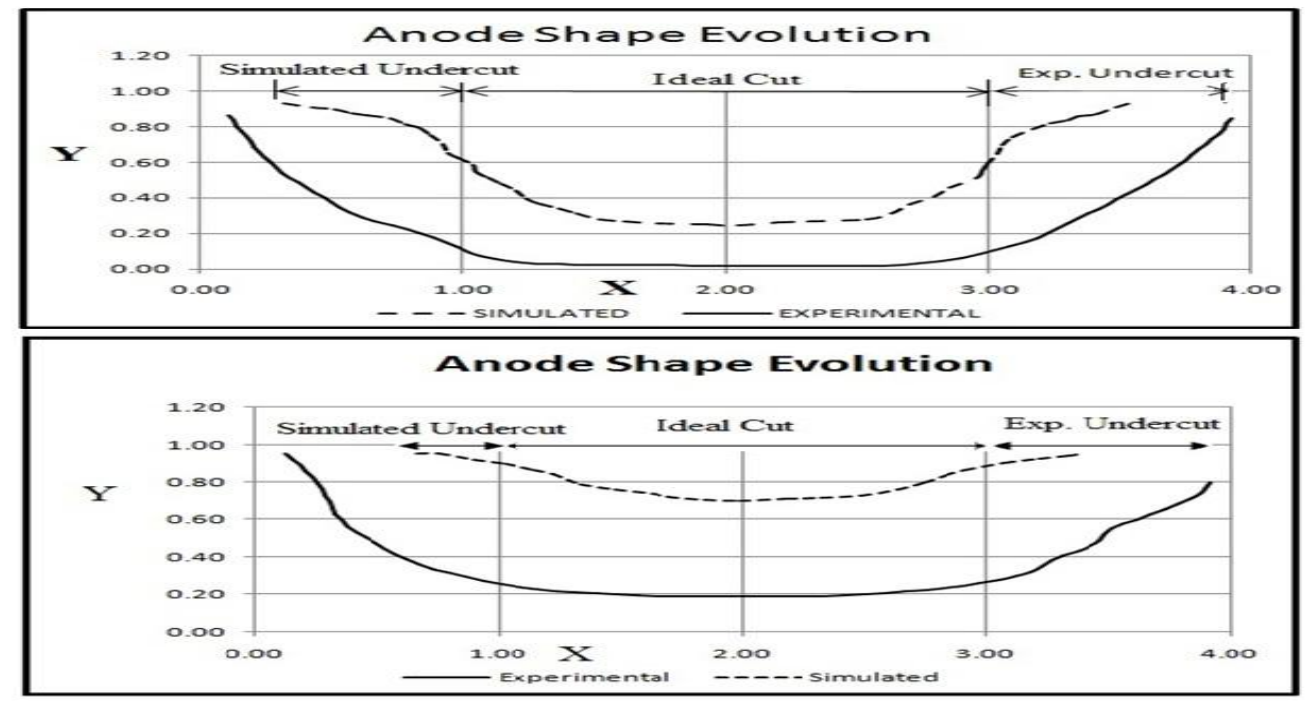

Fig. 9. Comparision Between Simulated and Experimental Anode Shape Profie (a) Voltage $=12 \mathrm{~V}$, IEG = $0.2 \mathrm{~mm}$ (b) Voltage $=10 \mathrm{~V}, \mathrm{IEG}=0.2 \mathrm{~mm}$; Concentration $40 \mathrm{gm} / \mathrm{Lit}$.

The comparison of the anode shape profile obtained through experimental shape and the simulated model is shown in fig 9. To validate the simulation results, the shape evolution process is observed experimentally. The experimental results shows that the hole has been formed which is not through uniform. It indicating that the current has mostly aggregated at the edge of the mask at the initial dissolution stage, the distribution of the current density within the machining surfaces shift away \& make the maximum in the current density from the edge of the hole toward its center as it given, which is qualitatively in good agreement with the simulation results. It is observed from fig 9 that the nature of the two profiles is approximately same but quantitatively deviation exists.

\section{Conclusions}

Simulation is a powerful tool for the design and improvement of electrochemical processes. Instead of an iterative tool and process design an FEM simulation can be an effective shortcut which reduces time and financial effort. Additionally, it visualizes the ECM process.

A set up has been developed for fabricating micro electrodes as well as machining micro holes and slots. The effect of key process parameters have been studied over the shape of the micro electrodes fabricated. The conclusions derived from the experiments are as follows:

- In this work, a two-dimensional finite element formulation using COMSOL Multiphysics of anode shape prediction model has been discussed. The designed work piece profile and the profile of the work piece evolved during experimentation agreed well.

- In the model only electrodynamics and mesh displacement are used. It seems that due to the very good electrolyte supply in the closed electrolytic free jet and the localization of the current density within it, ECMM can more easily be simulated than other applications of anodic dissolution.

- The geometry of the model is usefully to calculate ECMM point erosion up to about 40sec processing time.

\section{References}

[1]. Munda J, Bhattacharyya B (2008) Investigation into electrochemical micromachining (EMM) through response surface methodology based approach. Int J Adv Manuf Technol 35(7-8):821-832 .

[2]. Wang Li \&Wang Quandai \& Hao Xiuqing \& Ding Yucheng \& Lu Bingheng (2010), "Finite element simulation and experimental study on the through-mask electrochemical micromachining (EMM) 
process”, Int J Adv Manuf Technol 51:155-162 Published online: 13 April 2010 @ Springer-Verlag London Limited 2010.

[3]. V.K. Jain - Y. Kanetkar - G.K. Lal (2005) current attack and stagnation zones in electrochemical drilling, Int J Adv Manuf Technol (2005) 26: 527-536 Received: 29 November 2003 / Accepted: 20 May 2004 / Published online: 13 April 2005@ Springer-Verlag London Limited 2005

[4]. A.L. Krylo (1968), The Cauchy problem for Laplaces equation in theory of electrochemical processing of metal, Soviet Physics-Doklady 13 (1968) 15.

[5]. H. Tipton,(1970) Calculation of tool shapes for electrochemical machining, Journal of the Electrochemical Society 117 (1970).

[6]. D.E. Collet, R.C. Hewson-Browne, D.W. Windle,(1970) A complex variable approach to electrochemical machining problems, Journal of Engineering Mathematics(1970) 29.

[7]. A.D. Davydov, V.M. Volgin, V.V. Lyubimov,(2004) Electrochemical machining of metals: fundamentals of electrochemical shaping, Russian Journal of Electrochemistry 40 (2004) 1438.

[8]. [8] G.M. Alder, D. Clifton, F. Mill (2000), A direct analytical solution to the tool design problem in electrochemical machining under steady state conditions, in: Proc. Instn. Mech. Engrs., vol. 214, 2000, p. 745 .

[9]. J. Deconinck(1992), Current Distributions and Electrode Shape Changes in Electrochemical Systems, Springer-Verlag, Berlin, Heidelberg/New York, 1992.

[10]. O.H. Narayanan, S. Hinduja, C.F. Noble(1986), The prediction of workpiece shape during electrochemical machining by the boundary element method, International Journal of Machine Tools \& Manufacture 26 (1986) 323.

[11]. G.A. Prentice, C.W. Tobias, Simulation of changing electrode profiles, Journal of the Electrochemical Society 129 (1982) 78.

[12]. J. Kozak (1998), Mathematical models for computer simulation of electrochemical machining processes, Journal of Materials Processing Technology 76 (1998)170.

[13]. J. Kozak (2001), Computer simulation system for electrochemical shaping, Journal of Materials Processing Technology 109 (2001) 354.

[14]. Jain VK, Panday PC (1981) Tooling design for ECM - a finite element approach. J Eng Ind Trans ASME 103:183-190

[15]. Jain VK (1980) An analysis of ECM process for anode shape prediction. Ph.D. Dissertation, University of Roorkee

[16]. M. Hackert, G. Meichsner, A. Schubert (2008). Generating Micro Geometries with Air assisted Jet Electrochemical Machining. In Proceedings of the euspen 10th Anniversary International Conference, 2:420\{424, 2008. ISBN 978-0-9553082-5-3.

[17]. M. Hackert, G. Meichsner, A. Schubert (2009). Generating plane and microstructured surfaces applying Jet Electrochemical Machining. In Proceedings of Fifth International Symposium on Electrochemical Machining Technology, 2009.

[18]. M. Hackert, G. Meichsner, A. Schubert (2008). Simulation of the Shape of Micro Geometries generated with Jet Electrochemical Machining. Proceedings of the second European COMSOL Conference, 2008. ISBN 978-0-9766792-3-3

[19]. Jain, V.K. (2010), “Advanced Machining Processes”, Allied Publishers, N. Delhi. ISBN 81-77-64-2944

[20]. Jain, V. K., Gehlot Dileep,(2015) "Anode Shape Prediction in through mask Electrochemical Micromachining using FEM”, International journal of Machining Science \& Technology @ Taylor \& Francis Group, LLC(2015) ISSN 1091-0344 print/1532-2483 online.

[21]. Tipton (1964), H. The Dynamics of Electrochemical Machining, Proc 5th Int Machine Tool Design and Research Cant, 1964, 509522

[22]. Matthias et al,(2009) "Design of Electrochemical Machining Process by Multiphysics Simulation" COMSOL Multiphysics Conference. ol. III, G.T. Rado and H. Suhl, Eds. New York: Academic, 2009. 